\title{
Klebsiella pneumoniae Endocarditis
}

\author{
Shahla Roodpeyma ${ }^{1, *}$ \\ ${ }^{1}$ Department of Pediatric Cardiology, Shahid Beheshti University of Medical Sciences, Tehran, IR Iran \\ ${ }^{*}$ Corresponding author: Shahla Roodpeyma, Department of Pediatric Cardiology, Shahid Beheshti University of Medical Sciences, Tehran, IR Iran. Tel: +98-2122074087-98, Fax: +98- \\ 2122074101, E-mail: roodpeyma_shahla@yahoo.com
}

Received: May 8, 2014; Revised: June 28, 2014; Accepted: September 4, 2014

\begin{abstract}
Introduction: Gram negative bacteria including Klebsiella species are a rare cause of bacterial endocarditis in children. Klebsiella endocarditis usually needs a predisposing factor and has a poor prognosis.

Case Presentation: In this study a ten-year-old boy with ventricular septal defect, who was not immune compromised and acquired Klebsiella endocarditis is introduced. The patient had a favorable response to medical treatment and underwent a successful cardiac surgery. Discussion: Children with congenital heart disease are at risk of acquiring gram-negative endocarditis even if they have not had other predisposing factors.
\end{abstract}

Keywords: Children; Klebsiella endocarditis; Congenital Heart Disease

\section{Introduction}

Klebsiella species are part of the Enterobacteriaceae family. Members of this family are gram negative, non-sporeforming, and facultative anaerobes. Organisms of this family are widely distributed and are commonly found in the environment. There are three species in the Klebsiella genus; Klebsiella pneumoniae, Klebsiella oxytoca and Klebsiella granulomatis. They cause illness in human beings. Virulent Klebsiella strains produce highly mucoid colonies and have polysaccharide capsules, which protect them from phagocytosis, and help their adherence to tissues. Klebsiella pneumoniae (KP) may cause urinary tract infection (UTI), pneumonia, liver abscess, intraabdominal infections, and wound infection in healthy individuals. Klebsiella is a hospital acquired pathogen and can affect patients with underlying disease; however this organism may cause nosocomial infections through intravascular devices or invasive procedures. Klebsiella pneumoniae is the second gram-negative organism after Escherichia coli, which is responsible for bacteremia due to UTI. Klebsiella pneumoniae similar to other Enterobacteria species may cause systemic infections (1). In addition, KP can be a community-acquired pathogen. The pathogenicity of this organism is related to the production of a heat-stable and resistant enterotoxin. Underlying diseases such as diabetes mellitus, alcoholism, hemodialysis, malnutrition, malignancy, and previous implantation of intracardiac devices are risk factors for bacteremia and interaction between immune compromised host and virulent bacteria (2). Gram-negative bacterial endocarditis including KP is a rare phenomenon and makes up 5\% of all bacterial endocarditis. Primary KP endocarditis in an immune competent person is unlikely to occur, except in few instances such as, indwelling hard ware device, injection of illegal substances, previous history of hospital admission and having medical problems (3). Klebsiella accounts for $1.2 \%$ of native valve and $4.1 \%$ of prosthetic valve endocarditis. The aortic valve is most commonly involved, followed by mitral and tricuspid valves (4). Fortytwo percent of patients require valve replacement(5). Endocarditis due to $K$. oxytoca is very rare. Mortality due to Klebsiella endocarditis is higher than other types of endocarditis. In cases of delayed diagnosis, mortality rate are about 50\%, irrespective of wide spectrum antibiotic therapy (3). In a series of 35 cases with Klebsiella endocarditis, mortality rate was $49 \%$ (6). Patients who underwent valve replacement had a lower mortality rate than those who did not receive such treatment (7). Endocarditis due to intracardiac devices is rare and potentially fatal. Staphylococcus coagulase negative and Staphylococcus aureus are the most common organisms responsible for intracardiac-device infections. Gram-negative bacilli are rare in this kind of infection (3). All strains of KP are resistant to ampicillin. Therapeutic options for this infection include third or fourth generation cephalosporins, penicillin/ßlactamase inhibitor combination and aminoglycosides. Antibiotic treatment for six to eight weeks is necessary for cure. The bacteria can be grown on ordinary laboratory media (1). Cephalosporins and aminoglycosides are the most commonly prescribed antibiotics. The aim of the present study was to introduce an immune competent pediatric patient with congenital heart disease who acquired Klebsiella endocarditis.

Copyright (C) 2015, Pediartric Infections Research Center. This is an open-access article distributed under the terms of the Creative Commons Attribution-NonCommercial 4.0 International License (http://creativecommons.org/licenses/by-nc/4.0/) which permits copy and redistribute the material just in noncommercial usages, provided the original work is properly cited. 


\section{Case Presentation}

A ten-year-old boy was admitted to our ward due to a one-month history of fever and shivering. The patient was a known case of perimembranous ventricular septal defect (VSD). The parents had been advised about surgical closure of the defect but they were ignorant about this recommendation. There was no previous history of dental procedure or any other surgery. In physical examination, the general condition was bad and the patient was febrile, ill, toxic and anemic. Cardiac auscultation revealed a 4/6 harsh pansystolic murmur accompanied by thrill at the left lower sternal border. A faint diastolic flow rumble was audible at the apex. First and second heart sounds were covered by murmur. Fine crepitation rals were heard at the base of both lungs. Both the liver and the spleen were palpable at $2 \mathrm{~cm}$ below costal margins. Several petechia were noted at the nail beds of fingers.

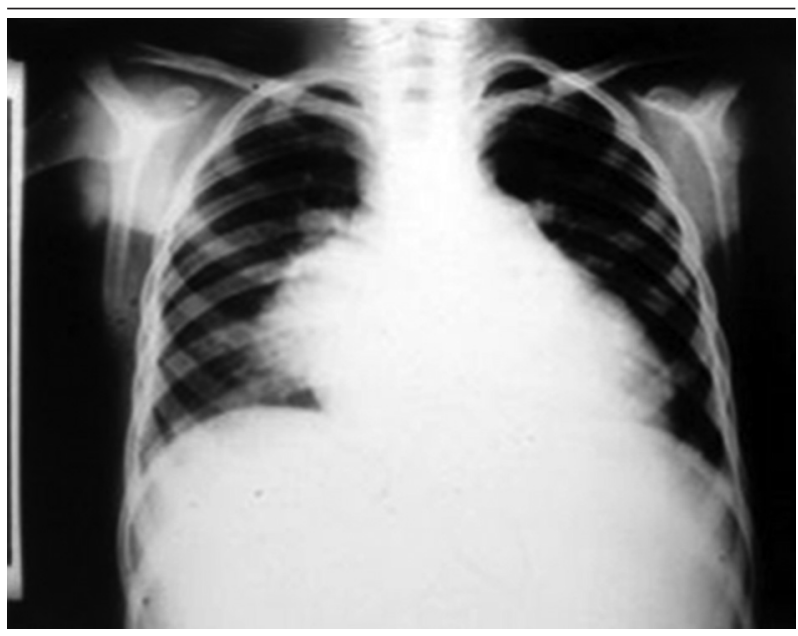

Figure 1. Chest X-Ray Showing Severe Cardiomegaly, Large Left Atrium and Left Ventricle, Prominent Pulmonary Artery Segment and Increased Pulmonary Blood Flow

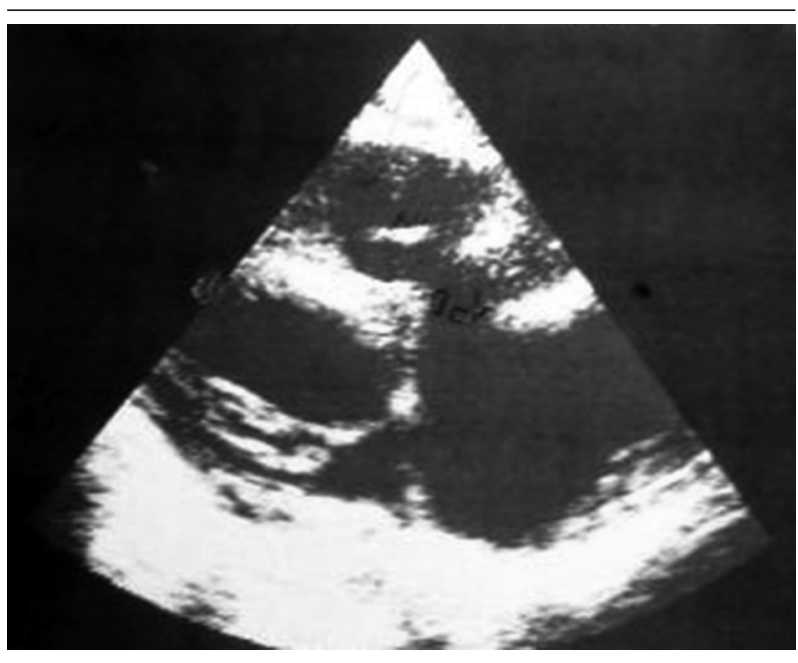

Figure 2. Echocardiogram Showing Large Left Atrium and Left Ventricle, Large Membranous Ventricular Septal Defect and a $5 \mathrm{~mm}$ Vegetation at the Right Ventricle
Chest $\mathrm{X}$ ray showed moderate to severe cardiomegaly consisting of a large left atrium, large left ventricle, prominent pulmonary artery segment and increased pulmonary blood flow (Figure 1). In electrocardiography, sinus tachycardia, normal axis and left ventricular hypertrophy were noted. Echocardiography showed a large left atrium, large left ventricle, large perimembranous ventricular septal defect, and a $5 \mathrm{~mm}$ vegetation at the right ventricular side of the ventricular septal defect (Figure 2). Cell blood count showed leukocytosis (WBC = 14600/ $\mu \mathrm{L}$ ) with polynuclear (89\%). Erythrocyte sedimentation rate (ESR) was $124 \mathrm{~mm} /$ hour. Three samples of blood were sent for microbiology culture. Treatment of congestive heart failure was started with digoxin, furosemide, aldactone and captopril. Diagnosis of bacterial endocarditis was suggested and empirical treatment with vancomycin and gentamicin was started. Congestive heart failure came under control yet there was no response to antibacterial treatment and the fever continued. At the 15th day of treatment 2 of 3 blood cultures yielded Klebsiella pneumoniae. The bacterium was sensitive to ceftriaxone and gentamicin. Therapeutic regime was changed to ceftriaxone and gentamicin. Four days after initiation of treatment with the new antibiotic, fever declined and then disappeared. With continuation of treatment, ESR declined to $26 \mathrm{~mm} /$ hour, cell blood count returned to normal levels and blood culture became negative. Treatment was continued for eight weeks. Repeated echocardiography showed organization of vegetation. After complete recovery of endocarditis, the patient was referred for cardiac surgery of VSD closure. The patient had a successful cardiac surgery and an uneventful postoperative course.

\section{Discussion}

Diagnosis of infective endocarditis (IE) in our patient was based on the two major Duke criteria, i.e. two separate positive blood cultures and evidence of vegetation on echocardiography. The underlying heart lesion in our patient was VSD. In the pediatric population bacterial endocarditis occurs among patients with congenital heart disease (CHD). In a five-year survey in Japan, 170 of 239 patients with bacterial endocarditis were children and 147 cases had CHD. Ventricular septal defect was the most common underlying heart disease, and Streptococcus viridans was the most common causative microorganism (8). Gram-negative bacteria account for $4-6 \%$ of bacteria isolated from children with endocarditis and $\mathrm{KP}$ is a rare organism. Ferreira et al. from Portugal reported on a fourmonth old infant with patent ductus arteriosus (PDA), admitted for bronchiolitis and acquired IE due to KP. After successful treatment of infection, the patient underwent surgical closure of PDA and recovered completely (9). In a research by Ifere et al. (10) from Nigeria, during an eightyears period (1981-1989) 32 children with IE were evaluated. Congenital heart disease was found in nine (28\%) cases. Positive blood culture was detected in 19 (58\%) patients. 
The most common organism was Staphylococcus. Klebsiella was detected in one case (11). Our patient showed a favorable response to treatment with ceftriaxone, and had a successful cardiac surgery. Results of a study about endocarditis due to gram-negative bacilli, on 28 patients, showed that two cases had KP. The overall mortality was $50 \%$. They found that surgery is usually needed after an effective four to six-week antimicrobial therapy (11). Beloborodova et al. (12) conducted a retrospective analysis on 39 patients who underwent cardiac surgery by cardiopulmonary bypass. The age of 28 adult patients varied from 44 to 58 years and that of 11 pediatric patients varied from four months to six years. Nine patients had IE at the time of operation. The results of the microbiological analysis showed high sensitivity of KP isolates to 3rd generation cephalosporin (12). The source of infection remained unknown in our patient. It is possible that his infection was community acquired. Recently the incidence of liver abscess with KP has increased in south-east Asia. This kind of $\mathrm{KP}$ is associated with metastatic abscess to other organs in $10 \%$ of cases. Rivero et al. (3) reported on a Filipino man with liver abscess and endocarditis. Liver CT scan revealed one large abscess in the right lower lobe, and transesophageal echocardiography showed large vegetation on the mitral valve, which resulted moderate to severe mitral regurgitation. Cultures from liver abscess grew KP, which was resistant to ampicillin but sensitive to all other antibiotics. The patient underwent cardiac surgery and the mitral valve was replaced with a prosthetic valve. The patient was discharged from the hospital after 41 days. Intravenous ceftriaxone had been continued for another six weeks at home. According to the authors, endocarditis as a complication of Klebsiella liver abscess had not reported before that time (4). In 2006, Chen et al. (4) reported on a case of community-acquired Klebsiella oxytoca endocarditis. The patient was a diabetic woman with chronic renal failure. Transthoracic echocardiography showed severe mitral and aortic regurgitation. Transesophageal echocardiography revealed large vegetation on the anterior leaflet of the mitral valve, and blood cultures were positive for Klebsiella oxytoca. This was the first case of community-acquired Klebsiella oxytoca endocarditis. The patient was treated with four weeks of intravenous cefazolin, and this was the first case report of community-acquired $K$. oxytoca endocarditis (4). Pai et al. (2) reported on a diabetic woman with dilated cardiomyopathy, congestive heart failure and left bundle branch block. Upgrading of her cardiac resynchronized pacemaker resulted in KP infection of pacemaker's lead and formation of mobile vegetation on the surface of right atrium's lead. Removing of the pacemaker and a sixweek treatment with intravenous cefotaxime cured the patient. The source of KP in this patient was unknown. It is possible that an occult hepatobiliary infection and hematogen spread was responsible for inoculation dur- ing implantation of the pacemaker (3). Endocarditis due to gram-negative bacteria is uncommon, although its incidence is increasing. Among gram-negative bacteria, Klebsiella species is a rare cause of endocarditis. In a review of literature (1945-1977), gram-negative bacterial endocarditis was reported in 348 patients, and only two cases were affected by Klebsiella (13). Data from Minneapolis microbiology laboratory showed that KP is the third cause of gram-negative bacteremia after Escherichia coli and Pseudomonas aeruginosa. In this sample, endocarditis occurred in $1.2 \%$ of Klebsiella bacteremia. The reason for such a low rate may be the weak adherence of Klebsiella to heart valves which is in contrast to the strong adherence of gram-positive bacteria (7). Searching for underlying disease and anticipating for complication are essential for management of patients. In conclusion, Klebsiella species is a rare but ominous cause of bacterial endocarditis and is associated with a high rate of complications and mortality. The presented patient may be the first case of Klebsiella endocarditis in children in the Iranian literature. His infection may have been acquired from the community, and the favorable response to treatment may be due to the fact that the patient was immune competent.

\section{References}

1. Donnenberg MS. Enterobacteriacease. Principle and practice of infectious diseases. In: Mandell GL, Bennett JE, Dolin R editors. Philadephia: Churchill Livingstone; 2010. pp. 2825-9.

2. Pai RK, Wall TS, Macgregor JF, Abedin M, Freedman RA. Klebsiella pneumoniae: a rare cause of device-associated endocarditis. Pacing Clin Electrophysiol. 2006;29(5):540-2.

3. Rivero A, Gomez E, Alland D, Huang DB, Chiang T. K2 serotype Klebsiella pneumoniae causing a liver abscess associated with infective endocarditis. J Clin Microbiol. 2010;48(2):639-41.

4. Chen JY, Chen PS, Chen YP, Lee WT, Lin LJ. Community-acquired Klebsiella oxytoca endocarditis: a case report. J Infect. 2006;52(5):e129-31.

5. Brouqui P, Raoult D. Endocarditis due to rare and fastidious bacteria. Clin Microbiol Rev. 2001;14(1):177-207.

6. Polk RE. pharmacotherapy: Infective endocarditis.Stanford, Connecticut: Appleton \& Lange; 1997.

7. Anderson MJ, Janoff EN. Klebsiella endocarditis: report of two cases and review. Clin Infect Dis. 1998;26(2):468-74.

8. Niwa K, Nakazawa M, Tateno S, Yoshinaga M, Terai M. Infective endocarditis in congenital heart disease: Japanese national collaboration study. Heart. 2005;91(6):795-800.

9. Ferreira PS, Rodrigues P, Peres A, Roque Do, Vale P, Casimiro A, Cunha F. Infective endocarditis complicating clinically silent patent ductus arteriosus. Acta Med Port. 2011;Suppl 3:605-10.

10. Ifere OA, Masokano KA. Infective endocarditis in children in the Guinea savannah of Nigeria.Ann Trop Paediatr. 1991;11(3):233-40.

11. Witchitz S, Regnier B, Witchitz J, Schlemmer B, Bouvet E, Vachon F. [Gram negative bacilli endocarditis ]. Pathol Biol (Paris). 1982;30(6 Pt 2):569-75.

12. Beloborodova NV, Kuznetsova ST, Popov DA, Bachinskaia EN, Vostrikov T. [Clinical experience with the treatment of severe nosocomial infections by inhibitor-protected 3rd generation cephalosporin cefoperazone/sulbactam]. Antibiot Khimioter. 2005;50(4):33-40.

13. Cohen PS, Maguire JH, Weinstein L. Infective endocarditis caused by gram-negative bacteria: a review of the literature, 1945-1977. Prog Cardiovasc Dis. 1980;22(4):205-42. 